\title{
Últimas aportaciones en la explicación de las crisis cambiarias: el caso de las crisis gemelas
}

\author{
Sofía García \\ Profesora e investigadora \\ Instituto Lawrence R. Klein \\ Centro Gauss, Universidad Autónoma de Madrid \\ sofia.garcia@uam.es \\ José Vicéns Otero \\ Catedrático de Economía Aplicada \\ Instituto Lawrence R. Klein \\ Centro Gauss, Universidad Autónoma de Madrid \\ jose.vicens@uam.es
}

\begin{abstract}
RESUMEN
A pesar de que han sido numerosos los episodios en los que algunas economías han experimentado simultáneamente colapsos en el mercado cambiario y suspensión de sus compromisos financieros externos, las aportaciones de la literatura financiera son recientes y exiguas. En el presente documento se pretende hacer una revisión de las aproximaciones teóricas que explican el estallido simultáneo de las crisis en ambos mercados, desde la corriente monetarista destacando las causas comunes, los mecanismos de contagio y los modelos que conceden un elevado rol a las expectativas de los agentes. Adicionalmente el artículo incluye una breve descripción de las principales aplicaciones empíricas relativas a esta tipología de crisis.
\end{abstract}


Palabras claves: Mercados financieros, crisis financieras, crisis de deuda, crisis cambiarias, crisis gemelas.

Código Jel: C10-F31

\section{INTRODUCCIÓN}

Durante las dos últimas décadas del siglo $\mathrm{xx}$, y especialmente en los años noventa, la financiación internacional ha sufrido un cambio radical y los capitales se han movido a una velocidad sin precedentes, producto de un proceso de liberalización creciente llevado a cabo en el seno de la llamada globalización financiera. Si bien tales movimientos traen consigo consecuencias positivas para los países receptores, también han tenido algunas connotaciones negativas, entre las que se encuentran la aparición, la intensificación y el contagio de las crisis financieras.

Un ataque especulativo en contra de la moneda de un país, una retirada súbita de los depósitos del sistema financiero, y una suspensión del pago de los compromisos financieros por parte de una nación, conllevan enormes costes para las sociedades en las que tienen lugar. Así, la pérdida de confianza por parte de los inversores en la política económica de un país, se mide finalmente, en términos de destrucción del empleo y de incrementos de los niveles de pobreza, dos de las secuelas más visibles de las crisis financieras.

En este sentido, conocer las causas que originan los colapsos financieros y los mecanismos de transmisión de las crisis se constituye en una herramienta imprescindible para evitar la aparición de nuevos episodios, y adicionalmente, para establecer lecciones al resto de economías que se encuentren en una situación de vulnerabilidad financiera o estén igualmente, sumergidas en una crisis.

La literatura teórica en torno a las causas que originan los episodios de crisis financieras es extensa y se ha ido adecuando a los cambios experimentados en los mercados financieros internacionales. De este modo, podemos encontrar por un lado, los modelos generacionales que intentan explicar los ataques especulativos en contra de las monedas (crisis cambiarias) y por otro lado, el conjunto de desarrollos teóricos que analizan las condiciones que derivan en un incumplimiento de las obligaciones financieras externas (crisis de deuda).

Más específicamente, los denominados modelos de primera generación señalan como la causa del ataque especulativo contra el tipo de cambio, la fuente de financiación de los desequilibrios en las cuentas fiscales; mientras que los modelos de segunda generación (o de expectativas autorrealizables), atribuyen el ataque especu- 
lativo a la existencia de distintas percepciones que conducen a la aparición de equilibrios múltiples, a los que la economía tiende dependiendo de las creencias y del comportamiento gregario de los agentes.

En relación al cuerpo de teorías que intentan explicar las razones que dan lugar a un incumplimiento del pago de los compromisos financieros, éstas señalan que un país incurre en una suspensión de pagos debido, fundamentalmente, a dos razones: a) porque no pueda cumplir con sus compromisos financieros (enfoque de Capacidad de Pago), o b) porque no esté dispuesto a hacerlo (enfoque de Coste Beneficio).

Por otro lado, la crisis financiera experimentada por los países asiáticos (19971998) constituyó un nuevo impulso para el desarrollo de los modelos que pretendían explicar las causas de las crisis monetarias y financieras. La mayoría de estos países presentaron, simultáneamente, problemas en su balanza de pagos y en el sistema financiero, dando origen a lo que la literatura denominó como «Crisis Gemelas»o «Twin Crisis». Estos modelos, catalogados como modelos de Tercera Generación — que analizan la interrelación entre la ruptura del sistema cambiario y la crisis del sistema financiero, han sido ampliamente debatidos y analizados en el campo de las finanzas internacionales a pesar de constituir un fenómeno relativamente reciente. Sin embargo, es importante destacar la existencia de un grupo alternativo de crisis gemelas: «las crisis cambiarias y las crisis sobre deuda soberana». Los desarrollos teóricos relativos a esta tipología de crisis son más recientes y tomaron como base el trabajo empírico realizado por Reinhart (2002), en el que demostró que el 84\% de las crisis de deuda que tienen lugar en los países emergentes están asociadas a la existencia de crisis en el sistema cambiario.

En este sentido, el presente documento tiene por finalidad hacer una revisión de las aproximaciones teóricas, así como de las principales aportaciones empíricas que intentan explicar el estallido de las crisis en ambos mercados con el objetivo de llevar a cabo en futuras investigaciones una aplicación empírica en el caso de los países latinoamericanos. Para ello el documento se ha estructurado como sigue: en el apartado II se realiza una revisión de la literatura sobre las crisis financieras, desde la óptica de los modelos generacionales; en el apartado III se profundiza en torno a las aportaciones teóricas que analizan las causas comunes, así como la anatomía del contagio entre las crisis de deuda y las de tipo de cambio; en el apartado IV se muestran los desarrollos teóricos recientes, bajo el enfoque coste beneficio, en los que conjugan el estado de los fundamentos económicos y las expectativas de los agentes como determinantes del estallido simultáneo de ambos colapsos financieros; y finalmente, en el apartado $\mathrm{V}$ se describen los principales hallazgos derivados del análisis empírico sobre las crisis gemelas en los países emergentes. 


\section{UNA BREVE REVISIÓN DE LOS MODELOS GENERACIONALES}

La literatura teórica sobre las crisis financieras y los incumplimientos en el servicio de la deuda soberana tratan de explicar, a grandes rasgos, el por qué se da una reducción repentina en el nivel de reservas internacionales o una huída masiva de capitales. La mayoría de los estudios conceden una elevada ponderación al rol que juegan las expectativas y coinciden en que la probabilidad de que ocurra una crisis se incrementa cuando un país atraviesa por una vulnerabilidad financiera.

Así, el primer grupo de modelos teóricos que explican las causas que originan una crisis de balanza de pagos están asociados con los Modelos de Primera Generación, donde la incompatibilidad de las políticas fiscal y monetaria con la política cambiaria produce un deterioro en los fundamentos económicos, que se convierte en la causa de las pérdidas de reservas internacionales por parte de la autoridad monetaria.

De modo más específico, Barberá y Blanca (2004), destacan que en esta clase de modelos la crisis ocurre porque las autoridades mantienen la paridad cambiaria fija y simultáneamente aplican una política monetaria expansiva con el fin de financiar el desequilibrio de las finanzas públicas. No obstante, dado que la demanda de dinero (en esta clase de modelos), es fija, la expansión de la oferta monetaria redunda en una reducción de las reservas internacionales. Así, la monetización del déficit fiscal es una política inconsistente con la de tipo de cambio fijo, ya que finalmente terminará por minar las reservas internacionales y debiendo, en consecuencia, abandonar el régimen cambiario.

El trabajo seminal de los modelos de primera generación lo constituye la obra de Krugman (1979). En este trabajo se destaca que ante un shock, si la economía se encuentra en una situación vulnerable, la aparición de la crisis es inevitable, a menos que las autoridades apliquen un conjunto de medidas que redunden en una mejora de la solvencia del país.

Las variables consideradas en esta tipología de modelos son útiles para explicar las causas de la crisis ocurrida en el primer quinquenio de la década de los 80 en Latinoamérica. En este sentido, Rodríguez (2001) indica que la crisis de la balanza de pagos experimentada por los países emergentes a principio de los ochenta, obedeció al compromiso de sus gobiernos de mantener fija la paridad cambiaria, unido a la falta de coordinación entre la política fiscal y la monetaria llevada a cabo por éstos.

No obstante, ante la imposibilidad de explicar las crisis financieras de algunos países (como la de México en 1994) a través del deterioro de los fundamentos eco- 
nómicos (modelos de la primera generación), surgió una corriente alternativa de investigación, los denominados Modelos de Segunda Generación.

Según Medina (2003), en esta tipología de modelos un cambio en las expectativas de los agentes sobre la posibilidad de que ocurra una crisis, puede conducir a un deterioro de las principales magnitudes macroeconómicas, precipitando en consecuencia el estallido de la crisis. Más aún, Barberá y Blanca (2004) señalan que una de las principales aportaciones de esta clase de modelos es que el sentido de la causalidad del colapso financiero no se da de manera unilateral, desde la situación de los fundamentos hacia las expectativas de los agentes, sino que la causalidad funciona en ambas direcciones, permitiendo en consecuencia, la existencia de equilibrios múltiples y la autoconfirmación de las expectativas.

Adicionalmente, Rodríguez (2001) resalta que la existencia de información imperfecta y asimétrica es de gran relevancia para entender los cambios repentinos en los sentimientos del mercado, que dan origen a un estallido de crisis de confianza. El primer aporte teórico en esta clase de modelos lo realizó Obstfeld (1996), quien dedujo que la existencia de equilibrios múltiples es posible y que un cambio en las expectativas puede originar un quebrantamiento de los fundamentos económicos, desencadenando en consecuencia, una crisis financiera.

Cabría destacar que algunos de los desarrollos teóricos elaborados bajo el enfoque Coste Beneficio ${ }^{1}$, relativos al incumplimiento de los compromisos financieros (crisis de deuda), también se apoyan en expectativas autorrealizables y en el comportamiento gregario de los agentes, pudiendo circunscribirlos, por tanto, en los denominados modelos de segunda generación (Calvo, 1988; Alesina-Prati-Tabellini, 1989; Cole y Kenoe, 2000).

Por otro lado, la crisis financiera experimentada por los países asiáticos (19971998) constituyó un nuevo impulso para el desarrollo de los modelos que pretendían explicar las causas de las crisis monetarias y financieras. Dado que la estabilidad macroeconómica era la constante para las principales economías de esta región (tasas elevadas de crecimiento económico y de ahorro, superávit en las cuentas fiscales, bajos niveles de inflación etc.), los modelos de primera generación no servían para

${ }^{1}$ La teoría coste beneficio establece que las naciones ponderan los costos y las ventajas de servir una deuda, eligiendo, de manera racional, renegociar un pasivo o simplemente dejar de pagar o repudiar una deuda en el caso de que esta decisión le genere mayores beneficios 
explicar el estallido de la crisis. Asimismo, la ausencia de cambios repentinos en la confianza de los agentes que empeorara los fundamentos de estas economías, igualmente, invalidaba las hipótesis contempladas en la segunda generación de modelos.

La mayoría de estos países presentaron, simultáneamente, problemas en su balanza de pagos y en el sistema financiero, dando origen a lo que la literatura denominó como «Crisis Gemelas» o «Twin Crisis». Estos modelos catalogados como modelos de Tercera Generación analizan la interrelación entre la ruptura del sistema cambiario y la crisis del sistema financiero.

Para las autoras Kaminsky y Reinhart (1998), las crisis financieras típicas tienen lugar cuando una economía entra en recesión habiendo experimentado previamente períodos de auge económico, caracterizados por una elevada expansión del crédito doméstico y por entradas masivas de capital. Así, la fase expansiva del ciclo crediticio puede presentar un punto de inflexión producto de la existencia de garantías sobre los depósitos, un insuficiente nivel de supervisión en el sistema financiero y el surgimiento de problemas de riesgo moral.

En cuanto al estallido simultáneo de la crisis en los mercados monetarios y financieros, éstas pueden desencadenarse por la existencia de causas comunes o a través de mecanismos de contagio. En este último caso, la relación causa efecto no es unidireccional, sino que en algunos casos una crisis bancaria puede conducir a un colapso en el sistema cambiario y viceversa.

En este sentido, según Alonso y Blanco (2004), las causas que pueden detonar un colapso en el tipo de cambio se sintetizan en:

- La creación de dinero - por parte de la autoridad monetaria-, con la finalidad de financiar el rescate generalizado del sistema financiero. El exceso de dinero en el mercado monetario puede conducir a un ataque especulativo en contra de la moneda.

Por otro lado, el contagio de una crisis cambiaria a una bancaria se puede explicar según los autores a través de los siguientes mecanismos:

- Una devaluación/depreciación puede debilitar la posición del sistema financiero, si éste se encuentra fuertemente endeudado en moneda extranjera.

- Un incremento de los tipos de interés, con la finalidad de defender la paridad, puede conducir a un empeoramiento de la cartera crediticia del sistema financiero. 
Finalmente los condicionantes comunes que pueden originar el estallido de las crisis en ambos mercados (cambiario y financiero) se pueden atribuir a los siguientes aspectos:

- La existencia de riesgo moral. El hecho de que las autoridades de una nación actúen como aval del sistema financiero, incentiva a que las instituciones financieras lleven a cabo estrategias de depósito e inversión con un elevado componente de riesgo. Las pérdidas de reservas internacionales a las que tendría que hacer frente el gobierno para preservar la estabilidad del sistema financiero, en el caso de que éste último no pueda afrontar sus pasivos, reduciría los grados de libertad del banco central para defender la paridad cambiaria. La soberbia obra de Krugman (1998), en la que explica, los detonantes de la crisis de Asia, se apoya en la hipótesis de la existencia de riesgo moral.

- La existencia de problemas temporales de liquidez en las entidades bancarias quedan exacerbados por cambios súbitos en los sentimientos de los inversores, en torno a la confianza en el sistema financiero. Estos cambios súbitos o repentinos de confianza pueden derivarse entre otros factores, de shocks externos (problemas de liquidez internacional, cambios en los tipos de interés, etc.) que apoyados en el desconocimiento sobre la solvencia de las instituciones domésticas puedan tener los inversores, provocarán una retirada súbita de los depósitos del sistema financiero y adicionalmente una pérdida de reservas internacionales, producto de la fuga de divisas. Al respecto, en el modelo desarrollado por los autores Chang y Velazco (1998) se demuestra que un shock externo puede profundizar la fragilidad o la vulnerabilidad del sistema financiero doméstico, desencadenando adicionalmente una ruptura en el sistema cambiario. De este modo, la financiación adicional por parte del banco central al sistema bancario para solventar los problemas de iliquidez temporal, puede ser empleada por los agentes para adquirir divisas. Dicha presión sobre el mercado cambiario terminará, finalmente, por minar la estabilidad cambiaria.

- La realización de procesos de liberalización financiera sin el establecimiento previo de adecuados mecanismos de supervisión y regulación. En el trabajo de Kaminsky y Reinhart (1998), en el que se analiza la relación entre las crisis cambiarias y las del sistema financiero, se comprobó empíricamente que los procesos de liberalización financiera intensifican las fases (expansivas y recesivas) de los ciclos crediticios, exacerbando las vulnerabilidades del sistema financiero. La continua financiación de parte de la autoridad monetaria a las instituciones crediticias culminaría por minar su capacidad para defender la 
paridad cambiaria, iniciando, adicionalmente, un círculo vicioso en el que las continúas devaluaciones empeorarán aún más la situación de las entidades crediticias altamente endeudadas.

\section{Crisis Gemelas: cambiarias y de deuda. CAUSAS COMUNES Y MECANISMOS DE TRANSMISIÓN}

Tal y como se mencionó en la introducción, el punto de partida que desencadenó esta nueva corriente de modelos lo constituye el trabajo llevado a cabo por Reinhart (2002), en donde demostró que el $84 \%$ de las crisis de deuda que tienen lugar en los países emergentes, están asociadas con la existencia de crisis en el sistema cambiario.

Más específicamente, la importancia de analizar ambos sucesos de manera simultánea quedó explícita en el número de crisis gemelas encontradas: 102, 34 y 24, en las aplicaciones empíricas que sobre esta tipología de crisis llevaron a cabo respectivamente Bauer, Herz y Volker (2007), Dreher, Herz y Volker (2004) y Herz y Tong (2004). Para analizar detalladamente este suceso, los autores detectaron los episodios de crisis cambiarias y de deuda que tenían lugar como sucesos aislados, separándolos de aquellas crisis en las que el colapso en los mercados de deuda y de tipo de cambio ocurría de manera simultánea.

Tabla 1. Número de crisis financieras. Evidencia empírica. Países emergentes

\begin{tabular}{|l|c|c|c|}
\hline AUTORES & $\begin{array}{c}\text { SÓLO DE DEUDA } \\
\text { Crisis de deuda sin } \\
\text { crisis cambiarias }\end{array}$ & $\begin{array}{c}\text { SÓLO DE TIPO DE } \\
\text { CAMBIO } \\
\text { (Crisis cambiarias sin } \\
\text { crisis de deuda) }\end{array}$ & $\begin{array}{c}\text { GEMELAS } \\
\text { (Crisis de deuda y } \\
\text { crisis cambiarias de } \\
\text { forma simultánea) }\end{array}$ \\
\hline Bauer, Herz y Volker (2007) & 229 & 145 & 102 \\
\hline Dreher, Herz y Volker (2004) & 246 & 145 & 34 \\
\hline Herz y Tong (2004) & 126 & 111 & 24 \\
\hline
\end{tabular}

En este contexto, Dreher, Herz y Volker (2004) realizaron un recorrido teórico en el que analizaron las posibles causas comunes, así como los efectos internos que podrían derivar en el contagio de un tipo de crisis a otra.

Los autores señalaron como posibles fuentes comunes que ocasionan, de forma simultánea, problemas en la balanza de pagos y en la financiación del presupuesto público de un país : 
- Un shock negativo (exógeno) sobre la demanda agregada que derive en una reducción de la actividad económica.

- En relación con la balanza de pagos: los modelos sobre crisis cambiarias señalan que el gobierno tendría grandes incentivos para abandonar la paridad e impulsar el crecimiento empleando una política monetaria expansiva. Adicionalmente, consideran que los especuladores que se comportan de una manera racional se anticiparán a la devaluación, efectuando huidas inesperadas de capital e incrementando, de este modo, la presión en el mercado cambiario. El aumento de los costes que supone el defender la paridad instará al gobierno a abandonarla, validando en consecuencia las expectativas de los especuladores.

- En relación con las finanzas públicas: las pérdidas en términos de producción y de empleo, ocasionadas por la recesión, incorporarán presiones sobre el presupuesto del gobierno, el que se vería en la obligación de elevar el gasto (fundamentalmente de transferencias sociales), incrementando los impuestos. Este hecho elevará la probabilidad de un incumplimiento en el servicio de la deuda, sobre todo en el caso de que a el gobierno se le impusieran restricciones para acceder a los mercados financieros internacionales o tuviese que emitir nueva deuda a tipos de interés muy elevados.

— Un incremento en los tipos de interés internacionales.

- Bajo perfecta movilidad de capitales si los deudores domésticos necesitaran refinanciar o emitir deuda, se verían en la necesidad de incrementar los tipos de interés doméstico. Los efectos adversos sobre el consumo y la inversión traerían una merma en la demanda agregada, desencadenando las mismas secuelas en la balanza de pagos y en el presupuesto público, descritas en el apartado anterior.

Cabe destacar que los autores señalan como única fuente común de vulnerabilidad financiera la producción de un shock internacional que pudiera mermar la producción interna de la economía. En cambio, existen desde el marco teórico enfoques adicionales (modelos de primera generación en lo que respecta a las crisis de tipo de cambio, y el enfoque de capacidad de pago, en el caso de las crisis de deuda) que otorgan importancia a la conducción de la política económica como eje primordial de análisis. En este sentido, tanto las decisiones de política económica que se hayan tomado en el pasado como el entorno globalizado en el que se encuentren insertas 
tales economías, determinarán el grado de vulnerabilidad financiera que éstas pudieran presentar en un momento determinado. En este caso, el marco teórico recomienda el seguimiento de un conjunto de indicadores macroeconómicos a través de los cuales se pueda evaluar cómo el desempeño de las políticas cambiaria, fiscal, monetaria, comercial y de endeudamiento podrían minar la generación de divisas del país y con ello, el cumplimiento tanto de los compromisos financieros como el resguardo de la estabilidad en el tipo de cambio.

Adicionalmente, en la obra de Jahjah y Montiel (2003) se analiza, desde una óptica interna de la economía, la interacción que existe entre el tipo de cambio y la propensión de las autoridades gubernamentales para incumplir el pago de los compromisos financieros externos, concluyendo, en el modelo teórico desarrollado, que tal relación no es exacta. Por un lado, el aumento del tipo de cambio puede disminuir la probabilidad de incumplimiento, siempre y cuando los beneficios derivados del aumento en la producción nacional superen los costes adicionales ocasionados por un mayor servicio de la deuda. En cambio, un empeoramiento en la percepción por parte de los acreedores, sobre el nivel de incumplimiento del gobierno, elevará la prima por riesgo demandada por éstos; el incremento en el pago de la deuda tenderá a mermar, los incentivos que tenga el gobierno para modificar al alza, el tipo de cambio.

En lo que respecta al efecto contagio entre las crisis objeto de estudio, los autores Dreher, Herz y Volker (2004) expusieron cuáles podrían ser los mecanismos de transmisión de una crisis de deuda a una cambiaria y viceversa.

- De la crisis de deuda a la crisis cambiaria:

- Considerando los efectos perjudiciales, en términos de comercio, producción y empleo (recesión) a los que se enfrenta un país al caer en suspensión de pagos, el abandono de la paridad puede ser la opción más loable. El intento por parte de los inversores de recuperar una porción de sus activos así como la política monetaria expansiva, que pudiera llevar a cabo el banco central de cara a estimular la economía y, finalmente, las presiones ejercidas por los especuladores crearán tensiones adicionales en el mercado cambiario que culminarían minando la paridad impuesta por la autoridad monetaria.

- De la crisis cambiaria a la crisis de deuda:

- Ante un ataque especulativo sobre el tipo de cambio, la autoridad monetaria puede decidir entre defender la paridad o devaluar. Cualquiera de las dos opcio- 
nes producirá grandes costos en términos de bienestar y afectará negativamente a la política presupuestaria y de endeudamiento del gobierno. En el caso de que la opción tomada fuera defender la paridad, la autoridad elevaría sostenidamente los tipos de interés doméstico. Este hecho, incrementaría la probabilidad de suspensión de pagos a través de dos vías. Por un lado, el aumento del costo del servicio de la deuda y por el otro, el efecto adverso sobre la demanda agregada, que reduciría los tributos recaudados empeorando la situación de las finanzas públicas. En el caso de que la autoridad monetaria optara por devaluar, las pérdidas en términos de credibilidad y de producción dificultarían el acceso a los mercados financieros internacionales aumentando la probabilidad de caer en suspensión de pagos.

- Una vía adicional de contagio la constituye el denominado «pecado original» ${ }^{2}$. A diferencia de los países desarrollados, la mayoría de las economías emergentes son incapaces de endeudarse a largo plazo en sus propias monedas. Este hecho, añade un plus a la vulnerabilidad de los países emergentes, ya que una devaluación supone un incremento en el servicio de la deuda externa y por tanto, un incentivo para incumplir con el pago a sus acreedores internacionales.

\section{Modelos Teóricos de Crisis Gemelas}

A diferencia del cúmulo de investigaciones orientadas a analizar aisladamente los colapsos en el mercado de deuda y en el tipo de cambio, recientemente se ha comenzado a elaborar un grupo de modelos teóricos que estudian la interrelación entre ambos tipos de crisis. En esta clase de modelos la decisión de devaluar y la de incurrir en suspensión de pagos no se toma de manera aislada, y se recaracterizan por presentar una función de bienestar — que el gobierno deberá maximizar-, unos parámetros de política - que representan la decisión de devaluar o no y de incumplir o no

${ }^{2}$ Un mayor detalle sobre el denominado pecado original de las economías emergentes se puede encontrar en las obras de Eichengreen, Hausmann y Panizza (2003): «Currency Mismatches, Debt intolerance and Original Sin: Why they are not the same and why it matters» y adicionalmente en Eichengreen, Hausmann y Panizza (2002): «Original Sin: The Pain, The Mystery, and the Road to Redemtion. 
con el pago del servicio de la deuda — y finalmente, una restricción presupuestaria que bien puede implicar inflación, devaluación o suspensión de pagos como mecanismos de financiación al déficit.

A continuación se expondrán los principales modelos existentes en la literatura teórica en relación con las crisis financieras acontecidas simultáneamente en los mercados monetarios y de deuda. Cabe destacar que la actualidad de estos desarrollos han servido de base para las posteriores aplicaciones empíricas en los mercados emergentes, detallados en el siguiente apartado.

\subsection{Modelo de Bauer-Herz-Volker (2003)}

Bauer et al (2003) señalan que la ocurrencia de ambos tipos de crisis (las cambiarias y las de deuda), no se deben exclusivamente al deterioro de los fundamentos económicos, sino que pueden desencadenarse producto de un cambio repentino en las expectativas de los agentes. Los autores destacan que las crisis con «expectativas que se autocumplen» se producen cuando los fundamentos económicos alcanzan un determinado nivel en el que tienen lugar equilibrios múltiples.

La función de bienestar que deberá maximizar el gobierno, en este modelo, viene dada por la siguiente ecuación:

$$
W \mathrm{t}=Y_{t}-\lambda E_{t}-\eta F_{t}-c T_{t}
$$

Donde:

$\mathrm{Y}_{\mathrm{t}}=$ Producto Interior Bruto del período $\mathrm{t}$.

$\mathrm{E}_{\mathrm{t}}=$ Costes totales de devaluación. Incluye costes fijos (pérdida de reputación en la capacidad de la autoridad monetaria), más costes variables (alza de tipo de interés).

$\mathrm{F}_{\mathrm{t}}=$ Costes totales de la suspensión de pagos. Incluye costes fijos (pérdidas en términos de comercio y de producción), más costes variables (prima por el riesgo que demandarían aquellos acreedores que estén dispuestos a refinanciar la deuda pendiente de pago).

$T_{t}=$ Impuestos requeridos para equilibrar las cuentas del estado.

Los autores señalaron que ante el esfuerzo por maximizar el bienestar, el gobierno se enfrenta básicamente a cuatro opciones: 
Tabla 2. Opciones de política económica

\begin{tabular}{|c|c|c|}
\hline & $\begin{array}{c}\text { No suspender el servicio } \\
\text { de la deuda }\end{array}$ & $\begin{array}{c}\text { Suspender el servicio } \\
\text { de la deuda }\end{array}$ \\
\hline No devaluar & No habrá crisis & Crisis de deuda \\
\hline Devaluar & Crisis Cambiaria & Crisis Gemelas \\
\hline
\end{tabular}

Fuente: Bauer et al (2003) página 4.

En el gráfico $\mathrm{N}^{\circ} 1$ los autores representan cuál sería la política óptima a llevar a cabo por el gobierno dadas las distintas combinaciones de nivel de endeudamiento y de la tasa de devaluación cambiaria, considerando el estado de los fundamentos económicos y las expectativas de los agentes privados. En dicho gráfico se distinguen dos zonas, una en la que existe sólo un equilibrio (Zonas 1-2-3 y 4) y otra en la que surgen equilibrios múltiples (Zonas 5-6 y 7). Las decisiones consideradas óptimas por el gobierno en cada una de las zonas se comentan a continuación:

Tabla 3. Decisiones de política económica óptimas

\begin{tabular}{|l|l|}
\hline Zona 1: & No produce crisis alguna. \\
\hline Zona 2: & Se origina, únicamente, una crisis cambiaria. \\
\hline Zona 3: & Se genera sólo una crisis de deuda. \\
\hline Zona 4: & Tiene lugar una Crisis Gemela. \\
\hline Zona 5: & $\begin{array}{l}\text { Existen dos posibles soluciones: o bien no ocurre ningún tipo de crisis o se da única- } \\
\text { mente una crisis de deuda. }\end{array}$ \\
\hline Zona 6: & $\begin{array}{l}\text { Existen dos posibles soluciones: o bien ocurre una crisis gemela o se da exclusiva- } \\
\text { mente una crisis cambiaria. }\end{array}$ \\
\hline Zona 7: & $\begin{array}{l}\text { Existen dos posibles soluciones: o bien no ocurre ningún tipo de crisis o se produce } \\
\text { una crisis cambiaria por el contagio de la crisis de deuda. }\end{array}$ \\
\hline
\end{tabular}

Fuente: Bauer, Herz y Kart (2003).

En las zonas 1-2-3 y 4 el gobierno efectúa una evaluación de los costes y los beneficios que le reportaría las distintas políticas en ambos mercados (monetario y de deuda), llevando a cabo aquellas que le reporten un mayor beneficio o un menor coste. Sin embargo, existe una banda denominada por los autores como «zona de crisis» (5-6 y 7) en la que las expectativas adquieren una gran relevancia (al igual que el estado de los fundamentos económicos), en la determinación de la política óptima a escoger por el gobierno. Así las expectativas que tengan los agentes sobre suspensión o 
no del servicio de la deuda que llevará a cabo el gobierno, siempre se validan. A diferencia de lo anterior, el rol que juegan las expectativas en materia de política cambiaria no es tan determinante. En la zonas 5 y 6 el nivel de los fundamentos será el único aspecto que considerar para que el gobierno decida según sea el caso, mantener la paridad o devaluar. Finalmente, en la zona 7 las expectativas sobre el incumplimiento de deuda se trasladan (contagian) a los ámbitos monetario y cambiario.

Gráfico 1. Combinación de expectativas de los agentes privados (de suspensión y no suspensión del pago de deuda)

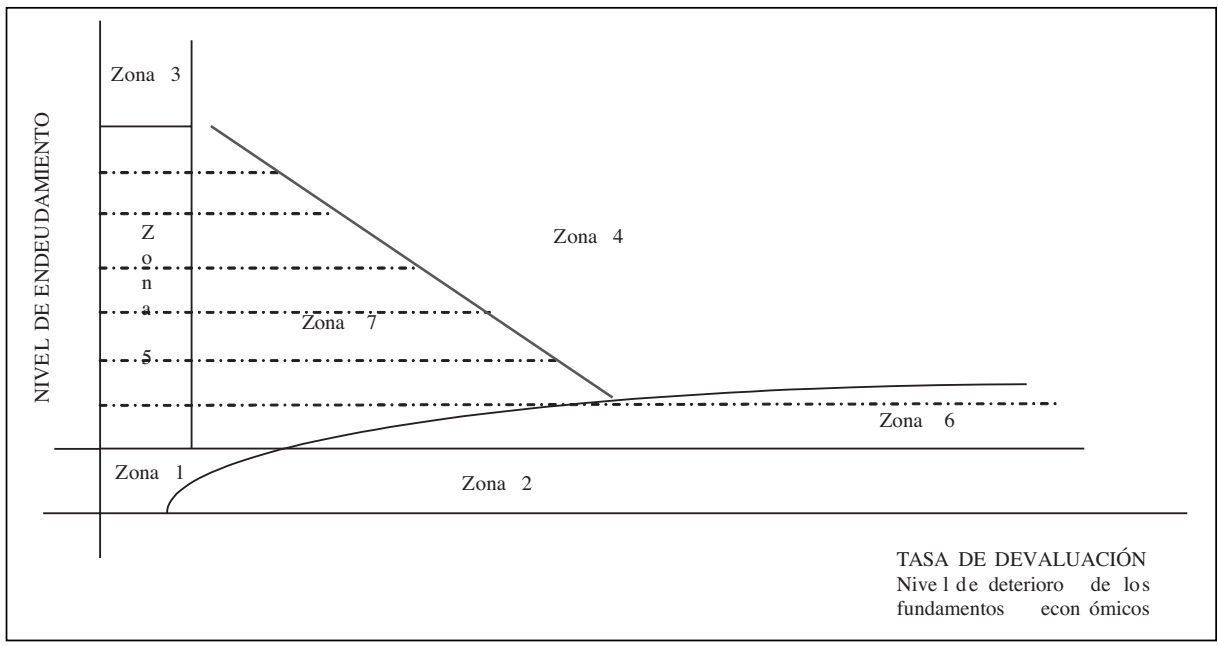

Adicionalmente a la consideración de la influencia ejercida por las expectativas de los agentes en el estallido de las crisis financieras, la principal aportación del modelo antes descrito, radica en la diligencia de la política económica para evitar el colapso en los mercados monetarios y de deuda. De este modo, la conducción de la economía hacia las zonas 5 y 7 (bajo nivel de endeudamiento y un nivel adecuado de los fundamentos económicos) podría minimizar (erradicando las expectativas perversas) el riesgo de crisis en los mercados. 


\subsection{Modelo de Herz y Tong (2004)}

Por su parte, Herz y Tong señalan la financiación del déficit como la fuente de vulnerabilidad financiera. Si el gobierno no tiene capacidad o no está dispuesto (por presiones institucionales o políticas) a incrementar la presión tributaria o a reducir el gasto, deberá financiar el déficit, incumpliendo parcialmente sus obligaciones financieras, o a través de un incremento de la cantidad de la oferta monetaria que impulsaría al alza el nivel de precios (lo que bajo el supuesto de paridad del poder adquisitivo, significa una devaluación del tipo de cambio en la misma magnitud), o finalmente, a través de una combinación de ambas (crisis gemelas). Por tanto, para financiar el déficit en ese modelo el gobierno emite moneda local (M), en pesos y las obligaciones externas $(f)$, en dólares.

El modelo teórico propuesto está basado en dos períodos. Donde:

$f_{m n}=$ deuda emitida en el momento $\mathrm{m}$, que deberá servirse en el momento $\mathrm{n}$.

$i=$ tipo de interés que paga el gobierno por la deuda emitida en moneda extranjera $i^{*}=$ tipo de interés libre de riesgo.

$g_{1}$ y $g_{2}=$ gasto público correspondientes a los momentos 1 y 2 .

Dada la paridad de poder adquisitivo, el tipo de cambio $E$ es igual al nivel interno de los precios $P$. Se asume $P$ igual a la unidad. Por tanto, $E_{1}$ y $E_{2}$ corresponden al tipo de cambio en los períodos 1 y 2 respectivamente.

El gobierno financia su gasto total en el período 1 (gasto total más servicio de la deuda) emitiendo deuda en moneda extranjera. Así, en el momento 1 el gobierno se enfrenta a la siguiente restricción presupuestaria:

$$
\frac{E_{1} f_{12}}{1+i}=E_{1} g_{1}+E_{1} f_{01}
$$

El gasto total del gobierno en el período 2 se financia por medio de incrementos de la masa monetaria (M2-M1), aumentos en los impuestos $\tau y$ (donde $y$ corresponde al producto nacional y $\tau$ a la carga impositiva) y a través de la suspensión de una fracción $(\eta)$ del servicio de su deuda externa. Con lo que la restricción presupuestaria del gobierno será en este período igual a: 


$$
(1-\eta)\left(E_{2} f_{12}+E_{2} f_{02}\right)+E_{2} g_{2}=M_{2}-M_{1}+E_{2} \tau y
$$

Bajo el supuesto de perfecta movilidad de capitales el equilibrio implicaría que el retorno sobre la tasa libre de riesgo $\left(i^{*}\right)$ y la tasa de interés $(i)$ que devenga la deuda externa, teniendo en cuenta un porcentaje $(\eta)$ de incumplimiento, deberán ser iguales.

$$
(1+i)(1-\eta)=(1+i)
$$

Por otro lado, la demanda de dinero vendría dada por la siguiente ecuación:

$$
M_{t}=\kappa E_{t} y \quad \text { para } t=1 \text { y } 2
$$

El gobierno tiene por objetivo minimizar los efectos de las crisis cambiarias y de deuda. Dado que la tasa de inflación/devaluación y la de incumplimiento se asumen igual a cero en el período 1, la función que resume las pérdidas financieras a las que se enfrenta el gobierno — dado el estallido de ambas crisis— puede ser escrita del siguiente modo:

$$
L=\frac{1}{2} \eta^{2}+\frac{\theta}{2} \varepsilon^{2}
$$

$\varepsilon=$ corresponde a la tasa de inflación y por tanto a la tasa de depreciación de la moneda.

$$
\varepsilon=\frac{E_{2}-E_{1}}{E_{2}}
$$

Simplificando la ecuación (1) y combinando las ecuaciones (2), (4) y (6) se tiene:

$$
\begin{gathered}
f_{12}=(1+i)\left(g_{1}+f_{01}\right) \\
\varepsilon \kappa y+\eta\left(f_{12}+f_{02}\right)=f_{12}+f_{02}+g_{2}-\tau y
\end{gathered}
$$


Si en el período 2 el gobierno no se compromete a cumplir con el pago de todos sus compromisos financieros tendrá que minimizar las pérdidas (ecuación 5), sujeto a la restricción dada por la función (8), con lo que se tendrá la siguiente condición necesaria:

$$
\frac{\theta \varepsilon}{\kappa y}=\frac{\eta}{f_{12}+f_{02}}
$$

Empleando la ecuación (9) para eliminar de (8), la tasa de suspensión de pagos bajo una crisis gemela sería igual a:

$$
\eta_{T W}=\frac{\theta\left(f_{12}+f_{02}\right)\left(f_{12}+f_{02}+g_{2}-\tau y\right)}{(\tau y)^{2}+\theta\left(f_{12}+f_{02}\right)}
$$

Al combinar las ecuaciones (7) y (10) se tiene que la tasa óptima de incumplimiento del gobierno depende del tipo de interés que paga por la emisión de su deuda. En perfecto equilibrio la tasa de incumplimiento esperada por el mercado debe ser igual a la tasa de incumplimiento que el gobierno encuentre óptima dadas las expectativas del mercado. El cumplimiento de esta condición generará la aparición de equilibrios múltiples.

La condición de arbitraje proveniente de la ecuación $\mathrm{N}^{\mathrm{o}} 3$ también relaciona la tasa de incumplimiento esperada por el mercado con el tipo de interés que devengan los títulos públicos.

$$
\eta_{M}=\frac{i-i^{*}}{1-i}
$$

Dadas estas condiciones, la economía puede tender a dos equilibrios. En ambos equilibrios el gobierno incumple una porción de sus obligaciones externas. Si el gobierno goza de credibilidad, el equilibrio al que tenderá la economía será aquél donde las pérdidas del gobierno resulten menores. Sin embargo, si el mercado interpreta que el gobierno incumplirá una porción mayor de su deuda, demandará una tasa de interés superior y la economía podría finalmente terminar en el equilibrio donde una tasa elevada de incumplimiento implicaría, adicionalmente, una mayor inflación y por tanto una mayor tasa de devaluación. 
Los autores suponen que en principio el gobierno siempre puede renegociar sus obligaciones en materia de deuda si las condiciones económicas lo justifican. Sin embargo, el gobierno se abstiene de emplear estas válvulas de escape por los costes fijos (exclusión de los mercados de capitales, caídas del PIB, entre otras) que ello puede implicar. De no existir tales costes, el gobierno siempre optaría por las crisis gemelas en lugar de escoger entre una de las dos crisis.

Al considerar los costes fijos $\left(C_{\eta}\right.$ y $\left.C \varepsilon\right)$, dado el estallido de ambas crisis, la función de pérdidas del gobierno vendría dada por la siguiente ecuación:

$$
L=\frac{1}{2} \eta^{2}+\frac{\theta}{2} \varepsilon^{2}+\lambda_{\eta} C_{\eta}+\lambda_{\varepsilon} C_{\varepsilon}
$$

$\operatorname{con} \lambda_{\eta}=1$ si $\eta \neq 0 ; \lambda_{\varepsilon}=1$ si $\varepsilon \neq 0$ y $\lambda_{\eta}=\lambda_{\varepsilon}=0$ en otro caso.

La política de financiación del presupuesto, el estado de los fundamentos económicos y las expectativas de los agentes son variables determinantes en la ocurrencia y el contagio de las crisis financieras. Si los agentes perciben que la tasa de suspensión a la que se compromete el gobierno no es creíble, demandarán una prima mayor por el riesgo asumido incrementando el servicio de la deuda. El consecuente deterioro en las finanzas públicas induciría al gobierno a suspender el pago de sus compromisos externos y adicionalmente, a financiar su gestión mediante inflación (devaluación).

El objetivo de analizar los dos modelos expuestos anteriormente radica en la creciente importancia que vienen adquiriendo las expectativas que puedan formularse los agentes económicos en torno a la credibilidad y el manejo adecuado de la política económica de un país. El desarrollo de nuevas tecnologías que reducen los problemas derivados de la información asimétrica, así como el comportamiento gregario de los agentes pueden exacerbar una situación de vulnerabilidad, conduciendo a la economía hasta un punto donde tenga lugar finalmente, la aparición de una crisis.

\section{UNA REVISIÓN SOBRE LAS APLICACIONES}

\section{A LAS CRISIS GEMELAS EN LOS MERCADOS EMERGENTES}

A continuación se presentan las aportaciones empíricas llevadas a cabo sobre el análisis de las crisis gemelas desde el punto de vista que nos atañe. Sobre cada una de las investigaciones se detallan los principales resultados obtenidos, la muestra seleccionada, la metodología empleada — resaltando de esta última la definición de 
crisis utilizada-, las variables explicativas que resultaron estadísticamente significativas y las herramientas estadísticas y econométricas empleadas en el análisis.

Como se ha señalado anteriormente, la investigación pionera de Carmen Reinhart (2002) en la que se analiza fundamentalmente, la capacidad del rating ${ }^{3}$ para predecir las crisis en el mercado de deuda ${ }^{4}$ y en el mercado monetario ${ }^{5}$ de 58 economías emergentes, analizadas desde 1970 hasta 1999, demostró que el 84\% de los incumplimientos en las economías emergentes están asociados con la aparición de crisis cambiarias. Lo contrario no es verdad. Menos de la mitad de las crisis cambiarias en las economías en desarrollo están vinculadas con los incumplimientos de los compromisos financieros. Para ello, la autora trabajó con cálculos de probabilidad condicional, analizando la probabilidad de que se diera una crisis de deuda/crisis cambiaria una vez transcurridos 24 períodos de haber tenido lugar un colapso en el tipo de cambio/crisis de deuda.

Los episodios de crisis cambiarias fueron analizados desde dos puntos de vista. Por un lado, se analizaron aquellas economías que presentaron una reducida senda en el crecimiento del nivel de precios, distinguiéndose por el contrario de aquéllas que presentaron una elevada inflación en el período de análisis.

En el primer caso, se aplicó un índice resultante del promedio entre la tasa de crecimiento del tipo de cambio y las reservas internacionales, empleando adicionalmente las desviaciones típicas del tipo de cambio y de las reservas internacionales, de forma tal que se igualaran las volatilidades de ambos (Kaminsky y Reinhart 1999). En cambio, para las economías inflacionarias se aplicó la observación directa del tipo de

${ }^{3}$ Según el Banco de España (1987) el rating constituye «una medida cualitativa del grado de riesgo crediticio de una deuda, es decir, de la capacidad del prestatario de devolver el principal y los intereses en las fechas establecidas en el contrato de emisión». Dichas calificaciones como lo señala Dalas (1993) sólo reflejan la opinión de las agencias que las emiten, en cuya determinación intervienen elementos cualitativos y cuantitativos.

${ }^{4}$ Un análisis exhaustivo sobre los determinantes macroeconómicos del rating soberano se puede encontrar en García y Vicéns (2006): «Factores condicionantes en la medición del riesgo soberano en los países emergentes».

${ }^{5}$ En la obra de Medina y Vicens (2006), bajo el enfoque econométrico, se realiza una selección de indicadores económicos útiles para predecir las crisis cambiarias en la región latinoamericana. 
cambio. En este caso, se consideró el estallido de una crisis cuando tenía lugar una devaluación de por lo menos un $25 \%$ en un mes dado, siempre que este porcentaje superara en al menos un 10\% la depreciación del mes anterior (Frankel y Rose 1996).

Por otro lado, para el análisis de los episodios de crisis de deuda se consideró como tal la suspensión del pago de los intereses y/o el capital de la deuda externa bancaria y la deuda externa contraída a través de la emisión de bonos.

Por su parte, Herz y Tong (2004) analizaron si la relación entre las crisis gemelas de deuda y de tipo de cambio, se debían a causas comunes o al contagio de un tipo de crisis a otra. Para ello estudiaron 74 economías emergentes durante el período muestral 1975-2001. La definición de crisis cambiaria empleada es al igual que en Reinhart (2002), la utilizada en el trabajo de Frankel y Rose (1996).

En relación a la crisis de deuda, se consideró que ésta tiene lugar cuando se produce una reprogramación de la deuda externa bajo la dirección del Club de París ${ }^{6}$. Los autores calcularon un modelo Probit (por máxima verosimilitud) y aplicaron el test de causalidad de Granger. Del análisis realizado, los autores encontraron fuertes evidencias de que el estado de algunos fundamentos económicos (como el ratio de reservas internacionales en término de las importaciones, el crecimiento del PIB y la inversión extranjera directa como porcentaje de la deuda externa) constituyen las causas comunes para el estallido simultáneo de ambas crisis. Finalmente, encontraron que existe una fuerte causalidad de Granger desde la crisis de deuda hacia las crisis cambiarias y una débil causalidad en el sentido Granger desde la crisis cambiaria hacia la de deuda.

Adicionalmente, Dreher, Herz y Volker (2004) investigaron aisladamente los determinantes de cada tipo de crisis empleando datos de panel con efectos fijos. Para medir el colapso en el tipo de cambio utilizaron la misma definición que Reinhart

${ }^{6}$ Es una reunión de acreedores (sin estatutos, cuotas, derechos de voto, ni sede propia) entre los que se encuentra los 19 países mas desarrollados del mundo, que se encargan de renegociar los vencimientos del principal y de los intereses concedidos a más de un año de plazo y cuyo prestatario puede ser un Estado soberano, una entidad pública con garantía del gobierno, un banco central o una deuda de naturaleza bilateral. En la actualidad el FMI y el Banco Mundial tienen un papel activo dentro del Club. Así el proceso de renegociación está sujeto a que se logre un acuerdo con FMI. Una vez alcanzado el acuerdo, si permanece el agujero financiero, entonces el club interviene a través del aplazamiento de los vencimientos de la deuda comercial del sector público, con la finalidad proporcionar alivio financiero requerido (Pérez, 2000). 
(2002) en el caso de economías no inflacionarias, definiendo por tanto un episodio de crisis, cuando el valor del índice superara al menos una desviación típica al promedio de éste. Por su parte el índice empleado para medir una crisis de deuda - calculado a través de la suma de los atrasos incurridos en el pago de los intereses y el capital correspondiente al servicio de la deuda-, debía superar al menos el $75 \%$ del servicio de la deuda para ser considerada como una crisis.

Del trabajo de Dreher, Herz y Volker (2004) se desprende que la única variable económica que vincula la existencia de causas comunes entre ambos episodios de crisis es la variable deuda pública. En relación a los mecanismos de contagio se encontró que el estallido de cualquiera de los dos tipos de crisis, incrementa la probabilidad de ocurrencia simultánea de la otra. Más aún, el test de Granger reveló la existencia de causalidad en ambas direcciones, inclusive hasta en el quinto retardo de las variables. Dicha relación positiva entre ambos sucesos de crisis se corroboró con la aplicación de otras técnicas econométricas (método generalizado de momentos, modelos bietápicos y trietápicos).

Más recientemente, Bauer, Herz y Volker (2007) analizaron 62 países de renta media (de entre 766 y 9.360 \$ que posean más de un millón de habitantes) durante el período 1975-2002 con el objetivo de demostrar que las crisis gemelas (cambiarias y de deuda), difieren de las crisis cambiarias y de deuda definidas como fenómenos aislados, en términos de causas, desarrollo y consecuencias. Así, empleando igualmente la definición de Frankel y Rose (1996) para el caso del tipo de cambio y considerando una reprogramación de los pagos externos o el incumplimiento con el servicio de la deuda como el estallido de crisis de deuda, lograron demostrar, aplicando modelos lineales de probabilidad, que cada tipo de crisis tiene lugar como producto de un conjunto de causas macroeconómicas particulares. Adicionalmente, comprobaron que el mecanismo de contagio y los efectos que se derivan de las crisis, pueden estar sesgados si no se considera cada una de ellas como un fenómeno en particular. Y finalmente, concluyeron que el análisis de las crisis como sucesos aislados mejora la eficiencia de los sistemas de alerta anticipada. 
Tabla 4. Resumen de la literatura empírica sobre crisis gemelas

\begin{tabular}{|l|l|l|}
\hline \multicolumn{1}{|c|}{ AUTORES } & \multicolumn{1}{|c|}{ MUESTRA } & \multicolumn{1}{c|}{ CONCLUSIONES } \\
\hline Reinhart (2002) & $\begin{array}{l}\text { 58 economías emergentes. } \\
\text { Período analizado: } \\
1970-1999\end{array}$ & $\begin{array}{l}\text { El 84\% de los incumplimientos de pagos en las economías } \\
\text { emergentes están asociados con crisis cambiarias. }\end{array}$ \\
\hline Dreher, Herz y Volker (2004) & $\begin{array}{l}80 \text { países en desarrollo. Perí- } \\
\text { odo analizado: 1975-2000 }\end{array}$ & $\begin{array}{l}\text { La única variable económica que vincula la existencia de } \\
\text { causas comunes entre ambos episodios de crisis es la } \\
\text { deuda pública. El estallido de cualquiera de los dos } \\
\text { tipos de crisis incrementa la probabilidad de ocurrencia } \\
\text { simultánea de la otra. }\end{array}$ \\
\hline Herz y Tong (2004) & $\begin{array}{l}\text { Período muestral: } \\
1975 \text { - 2001 }\end{array}$ & $\begin{array}{l}\text { Existen fuertes evidencias de que el estado de algunos } \\
\text { fundamentos económicos constituyen las causas comu- } \\
\text { nes para el estallido simultáneo de ambas crisis. Existe } \\
\text { una fuerte causalidad de Granger desde la crisis de deu- } \\
\text { da hacia las crisis cambiarias y una débil causalidad } \\
\text { desde la crisis cambiaria hacia la de deuda. }\end{array}$ \\
\hline Bauer, Herz y Volker (2007) & $\begin{array}{l}\text { 62 países de renta media. El } \\
\text { período corresponde a 1975- } \\
\text { 2002. }\end{array}$ & $\begin{array}{l}\text { Cada tipo de crisis tiene lugar producto de un conjunto de } \\
\text { causas particulares. El análisis de las crisis gemelas } \\
\text { como sucesos aislados mejora la eficiencia de los siste- } \\
\text { mas de alerta anticipada. }\end{array}$ \\
\hline
\end{tabular}

Fuentes: Elaboración propia.

Se pudo corroborar en las aplicaciones empíricas analizadas que el estado de algunos fundamentos económicos suponen los detonantes comunes del colapso simultáneo en ambos mercados. No obstante, se observa adicionalmente una cierta desviación con respecto a los fundamentos que las actuales teorías señalan como determinantes de las crisis gemelas objeto de estudio. En este sentido, para perfeccionar los sistemas de alerta anticipada deberían incluirse, en las futuras investigaciones sobre esta materia, variables proxys que representen por un lado, las expectativas de los agentes y por otro, el efecto que ocasionarían las variaciones del tipo de interés de las economías avanzadas sobre los mercados de deuda y de tipo de cambio en las economías emergentes. 


\section{Conclusiones}

Existe mayor evidencia empírica de que el estallido simultáneo de las crisis acontecidas en los mercados de deuda y de tipo de cambio se produce como una consecuencia del contagio entre ambas en lugar de obedecer al nivel que presentan determinados fundamentos económicos comunes. La mayoría de modelos estimados señalan la existencia de causas macroeconómicas particulares como detonantes aislados de cada tipo de crisis.

No se puede establecer una única dirección en el contagio de las crisis. Considerando el creciente papel que juegan las expectativas así como, el rol creciente de los sistemas de información, una vez haya tenido lugar el colapso en cualquiera de los mercados, se recomienda intentar revertir inmediatamente, cualquier expectativa que conduzca al estallido de una crisis gemela.

Si bien en la última década el porcentaje de crisis se ha reducido considerablemente, pudiéndose afirmar que en la actualidad los mercados monetarios y de deuda se encuentran en un período de estabilidad y calma, el incremento paulatino de los tipos de interés que caracteriza la coyuntura actual de las economías avanzadas —con la finalidad de mitigar los efectos de la inflación generada por el incremento sostenido de los precios del petróleo-, debe alertar sobre la posibilidad de nuevos episodios de crisis gemelas en los mercados emergentes. Se insta por tanto, a las autoridades gubernamentales de este tipo de países a mantener adecuados niveles de endeudamiento (menores a $100 \%$ del PIB) y a financiar su política fiscal con mecanismos quizás menos populares (incrementos de impuestos o reducción de gastos) de manera que se reduzca o revierta la percepción sobre una posible crisis por parte de los agentes.

Finalmente, de cara a prever futuros episodios de crisis deberá ahondarse en el estudio sobre aquellos mecanismos que condicionan la transmisión de las crisis, de forma tal que se disponga de sistemas de alerta anticipados más eficientes.

\section{Bibliografía}

Alesina, A.; Prati, A.; Tabellini, G. (1989): «Public Confidence and Debt Management: A model and a case study of Italia», NBER Working Paper Series, Working paper $\mathrm{N}^{\circ} 3135$, October.

Alonso, M.; Blanco, F. (2004): «Los modelos de crisis gemelas en el marco de la literatura 
sobre crisis monetarias internacionales» Información Comercial Española, ICE: Revista de economía, $N^{\circ} 816$, pp. 75.

Banco de España (1987): Circular No 22 del 29 de junio, Norma Cuarta de la Sección Primera, pp. $\mathrm{N}^{\mathrm{o}} 8$.

BARBERÁ, R.; BlANCA, M. (2004): «La interpretación de las crisis financieras a través de la literatura académica», Información Comercial Española (ICE), Revista de Economía, № 816, Julio-Agosto, pp. 9-21.

Bauer, Ch.; Herz, B.; VolKer, K. (2007):«Are twin currency and debt crises special?, Journal of Financial Stability, forthcoming.

- (2003): «The Other Twin Crisis: Currency and Debt», Review of Economics, Vol 54, No 3, pp. 248-267.

CaLvo, G. (1988): «Servicing the public debt: the rol of Expectations», The American Economic Review, Vol. No 78, pp. 647-661.

Chang, R.; Velazco, A. (1998): «Financial Crises in Emerging Markets: A Canonical Model», NBER Working Papers, Working paper $N^{\circ}$ W6606, June.

Cole, H.; Kenoe, T. (2000): «Self-Fulfilling Debt Crises». Review of Economics Studies, Vol. 67, Nº1, April, pp. 91-116.

Dallas, G. (1993): «Las agencias del calificación y el mercado de renta fija.», Papeles de Economía Española, № 54, pp. 286-295.

Dreher, A.; Herz, B.; Volker, K. (2004): Is there a causal link between Currency and Debt crises?, International Journal of Finance \& Economics, Vol. 11, Issue 4 , pp. 305-325.

Eichengreen, B.; Hausmann, R.; Panizza, U. (2003): «Currency Mismatches, Debt intolerance and Original Sin: Why they are not the same and why it matters», National Bureau of Economic Research, Working Paper N ${ }^{\circ}$ 10036, Octuber, pp. 1-62.

- (2002): «Original Sin: The Pain, The Mystery, and the Road to Redemtion», Inter-American Development Bank, Seminar papers, November, pp. 1-77

FrANKel, J.; Rose, A. (1996): «Currency crashes in emerging markets: An empirical treatment», Journal of International Economics, Vo. 41, No 3-4, pp. 351-368.

García, S.; Vicéns, J. (2006): «Factores condicionantes en la medición del riesgo soberano en los países emergentes», Estudios de Economía Aplicada, Vol. 24-1, Abril, pp. 245-272

Herz, B.; Tong, H. (2004): «The interaccions between Debt and Currency Crises- Common causes or contagion?», Universität Bayreuth, Diskussionspapier 17, Dezember, pp. 1-42.

Jahjah, S.; Montiel, P. (2003): «Exchange Rate Policy and Debt Crises in Emerging Economies», IMF Working Papers, Working Paper $\mathrm{N}^{\circ}$ WP/03/60, Washington D.C.

Kaminsky, G.; Reinhart, C. (1999): «The Twin Crises: The causes of Banking and Balance-OfPayment Problem», American Economic Review, Vol. 89, № 3, May, pp. 473-500.

- (1998): «Financial Crises in Asia and Latin America: Then and Now», American Economic Review, Vol. 88 Issue 2, May, pp. 444-448.

Krugman, P. (1998): «Whatever Happened to Asia?», WEB site: http://web.mit.edu/krugman/www/DISINTER.html 
Krugman, P. (1979): «A model of Balance of Payment Crises», Journal of Money, Credit and Banking, Vol. 11, № 3, August.

Krugman, P.; Obstfeld, M. (1997): «Economía Internacional». Teoría y Política, Tercera Edición, Editorial McGraw-Hill, Madrid.

Medina, E. (2003): «El uso de los modelos de elección discreta para la predicción de crisis cambiarias: el caso latinoamericano», Tesis doctoral. Universidad Autónoma de Madrid, Madrid.

Medina, E.; Vicéns, J. (2006): «Selección de indicadores adelantados de crisis cambiarias en Latinoamérica bajo un enfoque econométrico», Cuadernos de Economía, Vol. 29, № 79 , Enero-Abril, pp. 85-118.

Obsfeld, M. (1996): «Model of currency crises with self-fulfilling features», European Economic Review, No 40, pp. 1037-1048.

Pérez, C. (2000): «La arquitectura financiera internacional y el Club de París», Revista de Información Comercial Española, ICE. N ${ }^{\circ}$ 783, Enero-Febrero, pp. 33-50.

Reinhart, C. (2002): «Default, Currency Crises and Sovereign Credit Rating», The World Bank Economic Review, Vol. 16 No 2, pp. 151-170.

RodríGuez, G. (2001): «Globalización, Crisis Financieras y Dolarización». Ponencia presentada ante las Jornadas sobre Globalización realizada por el Instituto Universitario de Estudios Norteamericanos. Universidad de Alcalá. http://www2.uah.es/iuen/workingpapers/rodriguez.htm 
